\title{
NODA Award Recipients
}

Prepared by Sheena C. Williams

\section{Norman K. Russell Scholarship}

Roberta Stathis-Ochoa, Claremont Graduate School, 1982

Pamela Hall, University of South Florida, 1982

Cynthia Baxter, University of Vermont, 1983

Peggy Hayward, UCLA, 1983

JoAnn P. Sheeran, Bowling Green State University, 1984

Michael Allen Viney, University of Northern Colorado, 1984

Debra Julie Stogel, Northeastern University, 1985

Debora Joyce Clinton, Bowling Green State University, 1985

Jim Holmen, University of Vermont, 1986

Alex Duhamel, Michigan State University, 1987

Danielle Wilkshire, University of Maryland, 1987

Beth Adler, Bowling Green State University, 1988

Deborah Akers, Indiana University, 1988

Susan Bunker, Azusa Pacific University, 1988

Lori LeBahn, Missouri Southern State College, 1988

Diane Brimmer, Michigan State University, 1989

Betsey Lee Fuller, University of Maryland, 1989

Corinne Kowpak, University of Vermont, 1989

Elizabeth Rice, Ohio State University, 1989

J. Patrick Boyle, University of Pittsburgh, 1990

Sara Webster Schilling, Southern Illinois University - Carbondale, 1990

Shelly Ann Schwarzwalder, Kent State University, 1990

Donald Anthony Stenta, University of Vermont, 1990

Mary Kacmarcik, Pepperdine University, 1991

Lisa Domino, Kent State University, 1991

Christopher Eshelman, Wichita State University, 1991

Patricia Muller, Indiana University - Bloomington, 1991

Christina Cook, University of Michigan, 1992

Stephanie Fuji Heath, Truckee Meadows Community College, 1992

Craig Mack, Bowling Green State University, 1992

Tammy Peterson, North Carolina State University, 1992

Shannan Fasold, Colorado State University, 1993

Terri Marshall, St. Mary's University, 1993

Michael Minjares, San Diego State University, 1993

Gary Morgan, University of South Carolina, 1993

Kathi Captain, Florida State University, 1994 
Christina Purcell, Peabody College of Vanderbilt, 1994

Carol Lundberg, Claremont Graduate School, 1994

Andrea Hemphill, Texas A\&M University, 1994

Margaret Anlinker, University of South Carolina - Columbia, 1995

Kristin Corbett, Florida State University, 1995

John Seabreeze, University of Maryland - College Park, 1995

Anita B. Walton, North Carolina State University, 1995

Amy Donahue, Clemson University, 1996

Dan Turner, Northern Illinois University, 1996

Alicia Dawn Caudill, University of South Carolina, 1996

Sara B. Thomas, Miami University of Ohio, 1996

Regina D. Graber, University of Arkansas, 1997

Mark B. Reed, Western Illinois University, 1997

Kevin D. Rome, University of Texas at Austin, 1997

Courtney Erin Collier, Florida State University, 1998

Chastity Gerhardt, Oklahoma State University, 1998

Meg Nowak, Boston College, 1998

Brett E. Kennedy, University of Maryland, 1999

Maria S. Diaz, Florida State University, 1999

Karla Jean Felker, University of South Carolina, 1999

Mary Beth Mercatoris, University of Texas at Austin, 2000

Sarah Schoper, Miami University of Ohio, 2000

Erin Reicks, Iowa State University, 2000

Cody Arvidson, University of North Texas, 2001

Kellie Johnson, Miami University of Ohio, 2001

Marcus Skjervem, Illinois State University, 2001

Jene Kapela, College of William and Mary, 2002

Christopher Wyckoff, University of South Carolina, 2002

Mitsu Narui, Bowling Green State University, 2002

Michelle Murphy, Azusa Pacific University, 2003

Maren Oates, Oregon State University, 2003

Tricia Seifert, University of Iowa, 2003

Lucy LePeau, Indiana University, 2004

Emily Bauer, Oregon State University, 2004

Marci Rockey, Illinois State University, 2004

Kristin Price, Oregon State University, 2005

Rachael Stimpson, Virginia Tech University, 2005

Jess Webb, University of St. Thomas, 2005

Victoria "Tori" Svoboda, University of St. Thomas, 2006

Erin Biddlecombe, Oregon State University, 2006

Meredith Malnar, Texas A\&M University, 2006 


\section{Outstanding Orientation Professional}

Jimmy Abraham, Mississippi State University, 1993

Fran Butler, University of Arkansas, 1994

Pam Horne, University of Michigan, 1995

Maureen Hurley, Boston University, 1998

Brenda McKenzie, Kent State University, 2000

Les Cook, University of Utah, 2001

Denise Rode, Northern Illinois University, 2002

Ross Plasters, University of North Carolina - Greensboro, 2003

Cynthia Hernandez, Texas A\&M University, 2004

Jeb Branin, Southern Utah University, 2005

John "JB" Britton, Calvin College, 2006

\section{Outstanding New Professional}

Jeanine A. Ward, Clemson University, 1993

Michaelann Jundt, University of Washington, 1994

LeeAnn Melin, University of Minnesota, 1995

Melissa Trifiletti, Emory University, 1996

Christian Garcia, Millersville University, 1998

Dan Turner, Northern Illinois University, 1999

Mary Beth Mercatoris, University of Texas at Austin, 2000

Debbie Brawn, James Madison University, 2001

Patti Dulik, Purdue University, 2002

David Trillizio, Bradley University, 2003

Archie Cubarrubia, Northern Arizona University, 2003

Meredith Ray, Georgia Tech University, 2004

Drew Tinnin, University of Michigan - Ann Arbor, 2005

Marcus Langford, Oregon State University, 2006

\section{Outstanding Contributions to the Orientation Profession}

John Gardner, National Resource Center for The Freshman Year Experience, 1993 Lee Upcraft, Pennsylvania State University, 1994

Margaret Barr, Northwestern University, 1997

Bonita Jacobs, University of North Texas, 2000

Cathie Hatch, Bemidji State University, 2002

Richard Mullendore, University of Geogia, 2003

Jeanine Ward-Roof, Clemson University, 2004

Mary Jo Fabich, Illinois State University, 2005

Cindy Payne, Northern Arizona University, 2006 


\section{President's Award}

Ray Passkiewicz, Davenport College, 1993

Dick Mullendore, University of North Carolina, 1994

Gerry Strumpf, University of Maryland, 1995

Valerie Hodge, East Stroudsburg University, Pennsylvania, 1996

Michele McManus, University of North Carolina - Charlotte, 1997

Victor K. Wilson, University of Georgia, 1998

Bonita Jacobs, University of North Texas, 1999

Jeanine Ward-Roof, Clemson University, 2001

LeeAnn Melin, Northern Arizona University, 2002

Terese King, NODA Director of Membership Services (1999-2003), 2003

Jim Levi, Greek 101, 2004

Melanie Payne, Indiana University, 2005

Carren Martin, University of Wisconsin, 2006

Porter Martin, Attorney in Madison, WI, 2006

\section{Outstanding Research}

Beth Lingren, Southern Illinois University - Carbondale, 2002

Jeanine Ward-Roof, Clemson University, 2003

David Ortiz, University of Houston - Clear Lake, 2005

Greg Wolcott, San Jose State University, 2006

\section{Outstanding Service}

Tracy Forman, Texas A\&M University, 2004

J. J. Brown, University of Tennessee - Knoxville, 2005

Craig Mack, Boston University, 2006

\section{Outstanding NODA Intern}

Joshua Boschee, North Dakota State University, 2005

Kate Granholm, Illinois State University, 2005

Justin Brady, University of South Carolina, 2006

Kelly Rifenbark, Bowling Green State University, 2006

Jude Butch, Western Illinois University, 2006

Emily Williams, Jacksonville State University, 2006 


\section{Outstanding Network Chair}

Rob Anderson, University of Iowa (GLBTA Network), 2004

\section{Outstanding Regional Coordinator}

Larry R. Martin, California State - Fullerton (Region II), 2004

\section{Chancellor's Award}

Cheryl M. Hogle, University of New York - Plattsburgh, 1987 